\title{
The impact of financial incentives on participants' food purchasing patterns in a supermarket-based randomized controlled trial
}

Dana Lee Olstad ${ }^{1 *}$, David A Crawford ${ }^{2}$, Gavin Abbott ${ }^{2}$, Sarah A McNaughton², Ha ND Le ${ }^{3}$, Cliona Ni Mhurchu Christina Pollard ${ }^{5}$ and Kylie Ball ${ }^{2}$

\begin{abstract}
Background: The impacts of supermarket-based nutrition promotion interventions might be overestimated if participants shift their proportionate food purchasing away from their usual stores. This study quantified whether participants who received price discounts on fruits and vegetables (FV) in the Supermarket Healthy Eating for Life (SHELf) randomized controlled trial (RCT) shifted their FV purchasing into study supermarkets during the intervention period.

Methods: Participants were 642 females randomly assigned to a 1) skill-building ( $n=160), 2)$ price reduction ( $n=161)$, 3) combined skill-building and price reduction $(n=160)$, or 4$)$ control $(n=161)$ group. Participants self-reported the proportion of FV purchased in study supermarkets at baseline, 3- and 6-months post-intervention. Fisher's exact and $x^{2}$ tests assessed differences among groups in the proportion of FV purchased in study supermarkets at each time point. Multinomial logistic regression assessed differences among groups in the change in proportionate FV purchasing over time.

Results: Post-intervention, $49 \%$ of participants purchased $\geq 50 \%$ of their FV in study supermarkets. Compared to all other groups, the price reduction group was approximately twice as likely (RRR: 1.8-2.2) to have increased proportionate purchasing of FV in study supermarkets from baseline to post-intervention $(p<0.05)$.

Conclusions: Participants who received price reductions on FV were approximately twice as likely to shift their FV purchasing from other stores into study supermarkets during the intervention period. Unless food purchasing data are available for all sources, differential changes in purchasing patterns can make it difficult to discern the true impacts of nutrition interventions.
\end{abstract}

Trial registration: The SHELf trial is registered with Current Controlled Trials Registration ISRCTN39432901, Registered 30 June 2010, Retrospectively registered (http://www.isrctn.com/ISRCTN39432901).

Keywords: Supermarket, Food purchasing, Fruits and vegetables, Price reductions, Randomized controlled trial

\footnotetext{
*Correspondence: dana.olstad@ucalgary.ca

${ }^{1}$ Department of Community Health Sciences, University of Calgary, 3280

Hospital Drive NW, Calgary T2N 4Z6, Canada

Full list of author information is available at the end of the article
} 


\section{Introduction}

Supermarkets represent an important environment for influencing food selection and consumption given that most of the household food budget is spent there, and their significant power and control over the global food supply [1]. This power can be readily leveraged to negative, or alternatively, to more health-promoting ends because of the vast array of both healthy and unhealthy foods supermarkets offer [2]. Food prices are a key driver of food purchasing behaviors [3, 4]. The relative prices charged by supermarkets for healthy and unhealthy foods may therefore be one means through which supermarkets influence food purchasing and consumption [5]. Indeed, increased availability of low-cost, ultra-processed, energydense foods may be one reason why diet quality has declined in recent decades $[1,6]$.

Given evidence that supermarket pricing influences food purchasing [4], several supermarket-based randomized controlled pricing interventions have been conducted, with collective findings suggesting that price discounts on targeted healthier foods can increase their purchase [7-11]. The Supermarket Healthy Eating for Life (SHELf) randomized controlled trial (RCT) examined the impact of $20 \%$ price reductions on fruits and vegetables (FV) and healthier beverages, skill-building, and a combined price reduction + skill-building intervention on food purchasing within a single supermarket chain [7]. Purchasing outcomes showed that in the immediate postintervention period, the price reduction group increased purchasing of FV, while the combined group increased purchasing of fruit. However, findings from a process evaluation revealed that some participants randomized to receive price discounts reported having shifted their FV purchasing from other stores into study supermarkets to take advantage of discounts offered during the intervention period [12]. Previous supermarket-based RCTs have acknowledged that displacement of food purchasing might occur in pricing interventions [8], however to our knowledge no studies have confirmed that it does occur, nor examined its potential impact on study findings. Displacement of food purchasing is a concern because it may mask the true effectiveness of interventions. Thus, an apparent increase in FV purchasing within study supermarkets might actually represent a shift of FV purchasing from other food stores into study supermarkets, while overall FV purchasing remains unchanged. If similar shifts do not occur in the control group, change in food purchasing may be overestimated. The purpose of this study was to quantify whether, and to what extent participants who received price discounts on FV in the SHELf RCT may have shifted their FV purchasing from other stores into study supermarkets during the intervention period.

\section{Methods}

\section{Participants and setting}

Full methodological details of the SHELf RCT have been previously described [7, 13], and are briefly summarized here (Current Controlled Trials Registration ISRCTN39432901). The study was conducted amongst women living in one relatively advantaged, and one relatively disadvantaged neighborhood within Melbourne, Australia. These two neighborhoods were randomly selected from among those in the top (advantaged) and bottom (disadvantaged) tertiles of the Australian Bureau of Statistics' Socioeconomic Index for Areas [14] that were serviced by a Coles ${ }^{\ominus}$ supermarket (the second largest grocery chain in Australia) and located within $25 \mathrm{~km}$ of Deakin University. Two stores from within these neighborhoods were purposively selected for the study, and women who shopped at least once every 2 weeks within either of these target stores or any other Coles supermarket within a $5 \mathrm{~km}$ radius comprised the sampling pool. A random sample of 3000 eligible women received a mailout of a study recruitment package to their home address. A media release targeting local newspapers was also undertaken in the catchment areas to encourage additional participation. To be eligible to participate women had to shop regularly within Coles supermarkets (at least once every 2 weeks), hold or be willing to obtain a Coles store loyalty (FlyBuys) card, be between 18 and 60 years of age, the main household food shopper, able to speak, read and write in English, willing to have their sales data collected and analyzed, and be the only woman in their household enrolled in the study.

The study was conducted between May, 2011 and November, 2012. All procedures involving human subjects were approved by the Deakin University Faculty of Health Human Ethics Advisory Group (approval HEAG$\mathrm{H} 12 / 10$ ) and adhered to the principles of the Helsinki Declaration. All women provided written, informed consent prior to participating.

\section{Intervention}

Following completion of baseline surveys, participants were randomly allocated into one of four groups according to a computer-generated blocked randomization sequence produced and implemented by an independent statistician with a 1:1:1:1 participant allocation ratio: 1$)$ Control $(n=161), 2)$ Price reductions $(n=161)$, 3) Skillbuilding $(n=160)$, or 4 ) Combined price reductions and skill-building $(n=160)$. Allocation concealment was ensured via the secure storage of the randomization sequence separately from the participant database, which was only accessible to the data manager and statistician. Women enrolled in the price reduction arm received $20 \%$ discounts on all FV (including all varieties of fresh, 
canned, dried and frozen FV, excluding fruit juice, frozen french Fries and fried potatoes), and on low-energy carbonated beverages and water. The skill-building group received a set of eight mailed skill-building newsletters including information, activities and recipes to promote purchase and consumption of FV and healthier beverages. Individuals in the combined group received 20\% price discounts on FV and healthier beverages, along with all skill-building resources. The interventions took place over a 3-month period, with a further 6-month no-intervention follow-up phase. Women in the control group did not receive any interventions during the study period, and were asked to maintain their usual shopping habits.

\section{Data collection}

Participants completed self-report surveys at baseline (T1), post-intervention (T2, 3 months) and 6-months post-intervention (T3, 9 months). At baseline, participants reported sociodemographic characteristics including highest educational qualification, marital status and the number of children living in the home. To assess proportionate food purchasing, at all three time points participants reported the estimated proportion of their total groceries (How much of your total food and groceries are bought from Coles supermarkets?) and of their FV (How much of your fruit and vegetables are bought from Coles supermarkets?) they purchased at Coles (response options: less than a quarter, between a quarter and a half, between half and three quarters, more than three quarters, all of them). Changes in proportionate food purchasing from $\mathrm{T} 1$ to $\mathrm{T} 2$, and from $\mathrm{T} 2$ to $\mathrm{T} 3$ were estimated on the basis of these responses. For example, participants who reported purchasing $25-50 \%$ of their FV at Coles at $\mathrm{T} 1$ and $50-75 \%$ of their FV at Coles at T2 were deemed to have increased proportionate FV purchasing at Coles from $\mathrm{T} 1$ to $\mathrm{T} 2$.

\section{Statistical analyses}

Fisher's exact (for small expected cell sizes) and $x^{2}$ tests assessed differences among intervention groups in self-reported proportionate food purchasing at each time point. Multinomial logistic regression with robust standard errors was used to assess differences among intervention groups in change (classified as: increase, decrease, no change) in self-reported proportionate food purchasing from $\mathrm{T} 1$ to $\mathrm{T} 2$ and from $\mathrm{T} 2$ to $\mathrm{T} 3$. Data from all participants who completed any portion of the T1 ( $n=642 ; 100 \%$ of those randomized), T2 $(n=619$; $96 \%$ of those randomized) or T3 $(n=606 ; 94 \%$ of those randomized) surveys were included in these analyses, thus the sample size differed for each analysis.
All statistical tests were two-sided and the significance level was set at $p<0.05$. Stata software (version 13; StataCorp LP, TX) was used for all statistical analyses.

\section{Results \\ Descriptive characteristics}

The baseline sociodemographic characteristics of participants have been previously reported [7]. The sample was nearly evenly split according to neighborhood disadvantage, with $44 \%$ from low, and $55 \%$ from high socioeconomic status areas. Approximately half of the women were tertiary educated $(50 \%)$ and had at least one child living at home (53\%), and the majority were married (71\%) [7]. On average, between 71 and $80 \%$ of participants reported purchasing more than half of their total groceries at Coles, whereas just $45-49 \%$ of participants reported purchasing more than half of their total FV at Coles at all three time points (Table 1). A minority reported purchasing more than $75 \%$ of their groceries $(43-45 \%)$ and of their FV (25-28\%) at Coles at all three time points.

\section{Change in proportionate food purchasing}

Up to $50 \%$ of participants reported changing their proportionate food purchasing in study supermarkets during the study period (Table 2). From T1 to T2, individuals in the price reduction group were significantly more likely to report having increased proportionate purchasing of $\mathrm{FV}$ at Coles compared to individuals in the control (RRR: 2.15; 95\% CI: 1.26-3.68), skill-building (RRR: 1.94; 95\% CI: 1.143.32) and combined (RRR: 1.82; 95\% CI: 1.08-3.06) groups $(p<0.05)$. There were no other differences in change in proportionate food purchasing among groups from T1 to $\mathrm{T} 2$, or from $\mathrm{T} 2$ to $\mathrm{T} 3$.

\section{Discussion}

Supermarkets represent an important environment in which to intervene to improve population-level dietary behaviors. RCTs provide high quality data in support of causal inference, however even RCTs have limitations, particularly in the context of complex, real-world, population-level nutrition interventions $[15,16]$. In the SHELf RCT, individuals in the price reduction group were approximately twice as likely to report having increased proportionate purchasing of FV in study supermarkets from baseline to post-intervention, relative to all other groups. Qualitative comments from a prior process evaluation substantiated these findings, and suggested that these differential changes in FV purchasing were related to the financial incentive provided by the $20 \%$ price reductions [12]. SHELf is the first study, to our knowledge, to formally assess the patterning of proportionate food purchasing within the context of a supermarket-based RCT, albeit on the basis of self-reported data. 


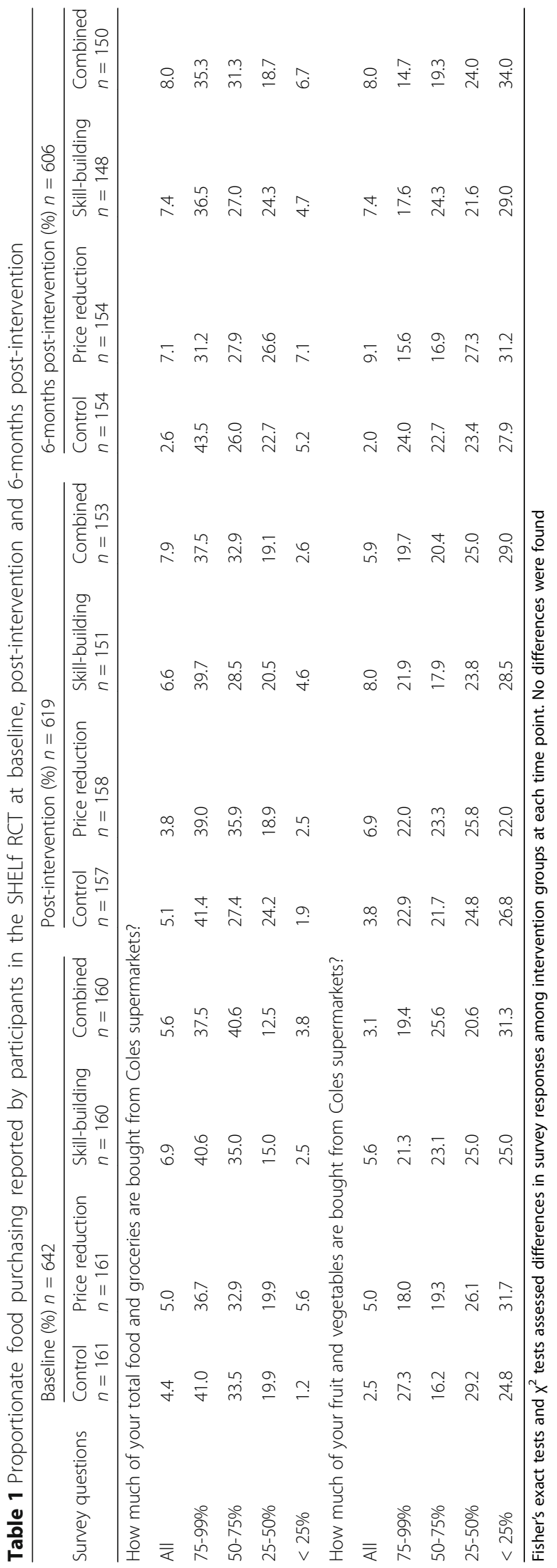


Table 2 Proportion of participants in the SHELF RCT who reported changing their proportionate food purchasing in study supermarkets

\begin{tabular}{|c|c|c|c|c|c|c|c|c|}
\hline & \multicolumn{4}{|c|}{$\begin{array}{l}\text { Change from baseline to } \\
\text { post-intervention (\%) } n=619\end{array}$} & \multicolumn{4}{|c|}{$\begin{array}{l}\text { Change from post-intervention to } 6 \text {-months } \\
\text { post-intervention (\%) } n=606\end{array}$} \\
\hline & $\begin{array}{l}\text { Control } \\
n=157\end{array}$ & $\begin{array}{l}\text { Price reduction } \\
n=158\end{array}$ & $\begin{array}{l}\text { Skill-building } \\
n=151\end{array}$ & $\begin{array}{l}\text { Combined } \\
n=153\end{array}$ & $\begin{array}{l}\text { Control } \\
n=154\end{array}$ & $\begin{array}{l}\text { Price reduction } \\
n=154\end{array}$ & $\begin{array}{l}\text { Skill- building } \\
n=148\end{array}$ & $\begin{array}{l}\text { Combined } \\
n=150\end{array}$ \\
\hline \multicolumn{9}{|c|}{ Change $^{a}$ in proportion of total food and groceries bought from Coles } \\
\hline Increase & 18.5 & 23.9 & 18.5 & 21.7 & 19.7 & 19.0 & 19.7 & 19.1 \\
\hline No change & 58.0 & 58.5 & 56.3 & 54.6 & 53.3 & 50.3 & 61.3 & 57.8 \\
\hline Decrease & 23.6 & 17.6 & 25.2 & 23.7 & 27.0 & 30.7 & 19.0 & 23.1 \\
\hline \multicolumn{9}{|c|}{ Change $^{a}$ in proportion of fruits and vegetables bought from Coles } \\
\hline Increase & 19.1 & 35.2 & 20.5 & 23.0 & 22.4 & 21.6 & 21.1 & 19.1 \\
\hline No change & 58.0 & 49.7 & 56.3 & 59.2 & 55.3 & 43.1 & 53.5 & 57.1 \\
\hline Decrease & 22.9 & 15.1 & 23.2 & 17.8 & 22.4 & 35.3 & 25.4 & 23.8 \\
\hline
\end{tabular}

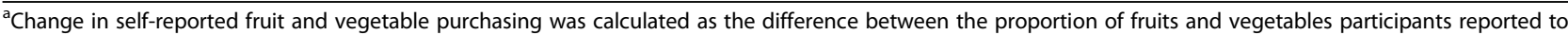
have purchased in study supermarkets at $\mathrm{T} 2$ compared to $\mathrm{T} 1$, and in the proportion of fruits and vegetables participants reported to have purchased in study supermarkets at T3 compared to T2

While RCTs are undoubtedly the gold standard of research design, collecting data pre- and postintervention, without measuring what happens in between, risks misstating the true impact of interventions on outcomes [15]. The current analysis was motivated by responses provided during a prior process evaluation of the SHELf RCT [12], and supported those earlier findings. When food purchasing data are not available from all sources, differential changes in proportionate food purchasing between randomized groups, such as those documented here, can make it difficult to discern the true impacts of nutrition interventions. This is because it becomes unclear to what extent measured changes in food purchasing are real, or merely represent shifts in food purchasing from other stores into study-associated venues, while overall purchasing remains constant. Notably, the Healthy Incentives Pilot rebate program also found evidence to suggest participants randomized to receive rebates may have partially shifted their $\mathrm{FV}$ purchases into participating retailers to maximize rebate earnings [17].

The current findings appear to be the result of two factors operating in tandem. First, the intervention and comparison conditions were somewhat asymmetric in terms of their perceived benefits to participants. Participants who received price reductions had a strong financial incentive to displace their FV purchasing from other stores into study supermarkets, whereas those in the control and skill-building groups did not [12]. Second, purchasing data were only collected within Coles supermarkets, and proportionate FV purchasing was low at Coles at all three time points (i.e. less than half of participants reported purchasing $\geq 50 \%$ of their FV at Coles, with only one-quarter purchasing $\geq 75 \%$ of their FV at Coles at all three time points). It is likely to be the co-occurrence of these circumstances that proved problematic. For instance, had complete purchasing data for participants been available for all venues in which they shopped, any asymmetries in the nature of the intervention and comparison conditions would have been relatively immaterial. Conversely, had the price reduction groups not had a strong incentive to displace their food purchasing, our inability to measure purchasing in all venues should not have posed a substantial limitation because randomization should have ensured similar proportions of participants changed their food purchasing behaviors over time.

It is not clear why a similar displacement of proportionate FV purchasing into study supermarkets was not evident in the group that received a combination of price reductions and skill-building. This result is, however, consistent with the different food purchasing and consumption behaviors exhibited by the price reduction and combined groups throughout the SHELf study [7]. This finding additionally suggests that while differential displacement of food purchasing may occur in some supermarket-based interventions, it is not necessarily inevitable, and should therefore be quantified and not assumed. It is also unclear why the change in self-reported proportionate FV purchasing in the price reduction group was not reflected in a similar shift in this group's total grocery purchasing, however we expect this may be because FV purchases comprised a relatively small proportion of participants' total food purchasing (i.e. participants purchased on average just $2.6 \mathrm{~kg} /$ week of FV) [7]. Finally, although $35 \%$ of those in the price reduction group reduced FV purchasing at Coles from T2 to T3 compared to an average of $22-25 \%$ in other groups, this decline was not statistically significant.

\section{Study limitations and strengths}

The current findings derive from self-reported data, however they are consistent with economic theories of 
human behavior, and are supported by regression analyses demonstrating directional correspondence between change in reported proportional, and objectively quantified FV purchasing (data not shown), as well as by qualitative comments provided during a prior process evaluation [12]. Moreover, identical questions were asked at all three time points, there was little missing data, and there is no reason to expect differential reporting of proportionate food purchasing over time.

\section{Implications}

The challenges associated with quantifying interventionassociated change in food purchasing described herein may be difficult to address given that modern environments offer ubiquitous access to food, and that incentives to alter food purchasing behaviors are inherent within the design of some supermarket-based interventions. The degree to which these and other potential biases arise will likely vary across studies according to factors such as the specific nature of each intervention, study location, and the participant population. Given that economic incentives are increasingly being deployed in an attempt to improve lifestyle behaviors, the current findings may also have broader relevance to other types of studies.

It is not possible to quantify to what extent shifts in food purchasing affected outcomes in the SHELf study. However, correspondence between purchasing and intake measures within the price reduction group provides reassurance that study-associated increases in fruit purchasing were not solely the result of displacement of proportionate fruit purchasing into study supermarkets. Moreover, that measured fruit purchasing increased in the combined group, a group in which significant displacement of proportionate FV purchasing was not reported is also instructive, as are qualitative comments from participants indicating that price discounts led them to purchase more FV overall. Authors of similar supermarket-based price interventions have employed sensitivity analyses [8], statistical adjustment [10], triangulation of measures $[10,11]$, and participant pledges to shop exclusively within study supermarkets [11] to avoid potential biases associated with displacement of food purchasing. The effectiveness of such strategies remains uncertain, however.

Study findings suggest that measurement of total food purchasing may be essential. These data could be obtained by asking participants to scan the bar codes of packaged foods brought into the home (e.g. analogous to the Neilsen Homescan Panel [18]) and to photograph unpackaged items, or by supplementing electronic purchasing data from study supermarkets with receipts from foods purchased elsewhere. However, just as individuals underreport food intake, households may also underreport their supermarket shopping $[19,20]$. These additional measures might also deter study participation due to increased participant burden, although in one study participants deemed collection of food receipts a useful tool in helping them to purchase healthier foods [21]. Development of new, technology-based integrated data collection methods may simultaneously improve the validity of the data collected, while reducing participant burden.

\section{Conclusions}

Supermarket-based interventions are a high priority given their substantial potential to improve food purchasing and intake at a population-level. Rigorous research designs are essential to ensure high quality data. RCTs are rightly regarded as the gold standard of research design, nevertheless they are not without limitations, particularly in the context of complex, real-world, population-level nutrition interventions $[15,16]$. In the SHELf RCT, compared to all other groups, participants who received price reductions on FV were approximately twice as likely to report having increased proportionate purchasing of FV in study supermarkets during the intervention period, potentially displacing a portion of their food purchasing from other venues during the intervention period. When food purchasing data are not available from all sources, differential changes in purchasing patterns such as those documented here can make it difficult to discern the true impacts of nutrition interventions. Future studies should attempt to proactively mitigate these potential biases, and where they do arise, should quantify their level and impact. Novel areas of inquiry could relate to development and testing of technology-based data collection methods, or to posthoc strategies to mitigate the impact of such biases.

\section{Abbreviations \\ FV: fruits and vegetables; RCT: Randomized controlled trial; SHELf: Supermarket healthy eating for life}

\section{Acknowledgements}

Not applicable

\section{Funding}

This study was funded by a National Health \& Medical Research Council (NHMRC) Project Grant, ID 594767. Coles ${ }^{\circledast}$ supermarkets and the National Heart Foundation of Australia provided in-kind support for the study. The study funders had no role in study design, in the collection, analysis, or interpretation of the data, or in writing this article. The contents of this paper are the responsibility of the authors and do not reflect the views of the NHMRC. DLO was supported by a Canadian Institutes of Health Research Fellowship and an Endeavour Research Fellowship. KB is supported by a National Health \& Medical Research Council Principal Research Fellowship, ID 1042442. SAM is supported by an NHMRC Career Development Fellowship Level 2, ID1104636 and was previously supported by an ARC Future Fellowship (2011-2015, FT100100581). CMP is supported by a Healthway Food Law, Policy and Communication to Improve Public Health Research Translation Grant.

\section{Availability of data and materials}

The datasets used during the current study are not publicly available due to their commercially sensitive nature, but are available from the corresponding 
author upon reasonable request and with permission from $\mathrm{Coles}^{\oplus}$ supermarkets.

\section{Authors' contributions}

DLO: analysed and interpreted the data, wrote the manuscript; DAC: designed the study, interpreted the data; GA: analysed and interpreted the data; HNDL: analysed and interpreted the data; SM, CNM, CP, DAC: designed the study, interpreted the data; KB: designed the study, supervised data collection, interpreted the data. All authors read and approved the final manuscript.

\section{Ethics approval and consent to participate}

All procedures involving human subjects were approved by the Deakin University Faculty of Health Human Ethics Advisory Group (approval HEAG-H 12/10) and adhered to the principles of the Helsinki Declaration. All women provided written, informed consent prior to participating.

\section{Consent for publication}

Not applicable

\section{Competing interests}

The authors declare that they have no competing interests.

\section{Publisher's Note}

Springer Nature remains neutral with regard to jurisdictional claims in published maps and institutional affiliations.

\section{Author details \\ 'Department of Community Health Sciences, University of Calgary, 3280 Hospital Drive NW, Calgary T2N 4Z6, Canada. ${ }^{2}$ Deakin University, Geelong, Australia, Institute for Physical Activity and Nutrition (IPAN), School of Exercise and Nutrition Sciences, Locked Bag 20000, Geelong 3220, Australia. ${ }^{3}$ Deakin University, Geelong, Australia, Deakin Health Economics, 221 Burwood Highway, Burwood, Victoria 3125, Australia. ${ }^{4}$ National Institute for Health Innovation, School of Population Health, The University of Auckland, Private Bag 92019, Auckland Mail Centre, Auckland 1142, New Zealand. ${ }^{5}$ School of Public Health, Faculty of Sciences, Curtin University, GPO Box U1987, Perth, Western Australia 6845, Australia.}

Received: 16 March 2017 Accepted: 17 August 2017

Published online: 25 August 2017

\section{References}

1. Hawkes C. Dietary implications of supermarket development: a global perspective. Dev Policy Rev. 2008:26(6):657-92.

2. Zenk SN, Powell LM, Rimkus L, Isgor Z, Barker DC, Ohri-Vachaspati P, Chaloupka F. Relative and absolute availability of healthier food and beverage alternatives across communities in the United States. Am J Public Health. 2014;104(11):2170-8.

3. An R. Effectiveness of subsidies in promoting healthy food purchases and consumption: a review of field experiments. Public Health Nutr. 2013;16(7): 1215-28.

4. Epstein LH, Jankowiak N, Nederkoorn C, Raynor HA, French SA, Finkelstein $E$. Experimental research on the relation between food price changes and food-purchasing patterns: a targeted review. Am J Clin Nutr. 2012;95(4):789-809.

5. Lee A, Mhurchu CN, Sacks G, Swinburn B, Snowdon W, Vandevijvere S, Hawkes C, L'Abbe M, Rayner M, Sanders D, et al. Monitoring the price and affordability of foods and diets globally. Obes Rev. 2013;14(Suppl 1):82-95.

6. Rehm CD, Monsivais P, Drewnowski A. Relation between diet cost and healthy eating index 2010 scores among adults in the United States 2007-2010. Prev Med. 2015;73:70-5.

7. Ball K, McNaughton S, Le H, Gold L, Ni Mhurchu C, Abbott G, Pollard C, Crawford D. Influence of price discounts and skill-building strategies on purchase and consumption of healthy food and beverages: outcomes of the supermarket healthy eating for life randomized controlled trial. Am J Clin Nutr. 2015;101(5):1055-64.

8. Ni Mhurchu C, Blakely T, Jiang Y, Eyles HC, Rodgers A. Effects of price discounts and tailored nutrition education on supermarket purchases: a randomized controlled trial. Am J Clin Nutr. 2010;91(3):736-47.
9. Phipps EJ, Braitman LE, Stites SD, Singletary SB, Wallace SL, Hunt L, Axelrod $\mathrm{S}$, Glanz K, Uplinger N. Impact of a rewards-based incentive program on promoting fruit and vegetable purchases. Am J Public Health. 2015;105(1): 166-72.

10. Waterlander WE, de Boer MR, Schuit AJ, Seidell JC, Steenhuis IH. Price discounts significantly enhance fruit and vegetable purchases when combined with nutrition education: a randomized controlled supermarket trial. Am J Clin Nutr. 2013;97(4):886-95.

11. Geliebter A, Ang IY, Bernales-Korins M, Hernandez D, Ochner CN, Ungredda T, Miller R, Kolbe L. Supermarket discounts of low-energy density foods: effects on purchasing, food intake, and body weight. Obesity (Silver Spring). 2013;21(12):E542-8.

12. Olstad DL, Ball K, Abbott G, McNaughton SA, Le HN, Ni Mhurchu C, Pollard C, Crawford DA. A process evaluation of the supermarket healthy eating for life (SHELf) randomized controlled trial. Int J Behav Nutr Phys Act. 2016:13:27.

13. Ball K, McNaughton SA, Mhurchu CN, Andrianopoulos N, Inglis V, McNeilly B, Le HN, Leslie D, Pollard C, Crawford D. Supermarket healthy eating for life (SHELf): protocol of a randomised controlled trial promoting healthy food and beverage consumption through price reduction and skill-building strategies. BMC Public Health. 2011;11:715.

14. Australian Bureau of Statistics. Census of population and housing. Australia 2006. http://www.abs.gov.au/AUSSTATS/abs@.nsf/DetailsPage/2033.0.55. 0012006?OpenDocument. Accessed 11 Apr 2015: Socio-Economic Indexes for Areas (SEIFA); 2006. Data only

15. Hebert JR, Frongillo EA, Adams SA, Turner-McGrievy GM, Hurley TG, Miller DR, Ockene IS. Perspective: randomized controlled trials are not a panacea for diet-related research. Adv Nutr. 2016;7(3):423-32.

16. Sanson-Fisher RW, Bonevski B, Green LW, D'Este C. Limitations of the randomized controlled trial in evaluating population-based health interventions. Am J Prev Med. 2007;33(2):155-61.

17. Olsho LE, Klerman JA, Bartlett SH, Logan CW. Rebates to incentivize healthy nutrition choices in the supplemental nutrition assistance program. Am J Prev Med. 2017:52(252):S161-70.

18. Nielsen. Nielsen Homescan Panel. https://www.homescan.com/panel/US/ EN/Login.htm. Accessed 13 Apr 2015.

19. Ransley JK, Donnelly JK, Khara TN, Botham H, Arnot H, Greenwood DC, Cade JE. The use of supermarket till receipts to determine the fat and energy intake in a UK population. Public Health Nutr. 2001;4(6):1279-86.

20. Burney J, Haughton B. EFNEP: a nutrition education program that demonstrates cost-benefit. J Am Diet Assoc. 2002;102(1):39-45.

21. Foley RM, Pollard CM. Food cent\$-implementing and evaluating a nutrition education project focusing on value for money. Aust N Z J Public Health. 1998;22(4):494-501.

\section{Submit your next manuscript to BioMed Central and we will help you at every step:}

- We accept pre-submission inquiries

- Our selector tool helps you to find the most relevant journal

- We provide round the clock customer support

- Convenient online submission

- Thorough peer review

- Inclusion in PubMed and all major indexing services

- Maximum visibility for your research

Submit your manuscript at www.biomedcentral.com/submit
) Biomed Central 